\title{
Economic Analysis of the CLIP Trials in India, Pakistan and Mozambique
}

\section{Jeffrey N Bone}

The University of British Columbia https://orcid.org/0000-0001-7704-1677

Asif Khowaja

The University of British Columbia

Marianne Vidler

The University of British Columbia https://orcid.org/0000-0002-7633-8812

Beth A. Payne

The University of British Columbia https://orcid.org/0000-0001-6150-8703

Mrutyunjaya B Bellad

KLE University https://orcid.org/0000-0003-0460-1439

Shivaprasad S Goudar

KLE University https://orcid.org/0000-0002-8680-7053

Ashalata Mallapur

BVVS S Nijalingappa Medical College and HSK Hospital and Research Centre

Khátia munguambe

Centro de Investigacao em Saude de Manhica

Rahat Qureshi

Aga Khan University https://orcid.org/0000-0002-3215-3445

Charfudin Sacoor

Centro de Investigacao em Saude de Manhica https://orcid.org/0000-0002-8724-0319

Esperança Sevene

Centro de Investigacao em Saude de Manhica

Geert Frederix

Universiteit Utrecht

Zulfiqar Bhutta

SickKids Research Institute https://orcid.org/0000-0003-0637-599X

Craig Mitton

The University of British Columbia https://orcid.org/0000-0001-7871-8977

Laura Magee

King's College London https://orcid.org/0000-0002-1355-610X

Peter von Dadelszen ( $\sim$ pvd@cw.bc.ca)

The University of British Columbia

\section{Research}

Keywords: CLIP, POM, YLL, arms, Pre-eclampsia.

Posted Date: August 25th, 2020

DOI: https://doi.org/10.21203/rs.3.rs-60194/v1

License: (c) (1) This work is licensed under a Creative Commons Attribution 4.0 International License. Read Full License 


\section{Abstract}

Background: The Community-Level Interventions for Pre-eclampsia (CLIP) Trials (NCT01911494) in India, Pakistan, and Mozambique (February 2014-7) involved community engagement and task-sharing with community health workers for triage and initial treatment of pregnancy hypertension. Maternal and perinatal mortality was less frequent among women who received $\geq 8$ CLIP contacts. The aim of this analysis was to assess the costs and cost-effectiveness of the CLIP intervention overall, and by POM visit frequency.

Methods: Included were all women enrolled in the three CLIP trials who had delivered with known outcomes by trial end. According to the number of POMguided home contacts received $(0,1-3,4-7,38)$, costs were collected from annual budgets and spending receipts, with inclusion of family opportunity costs in Pakistan. A decision-tree model was built to determine the cost-effectiveness of the intervention (vs. usual care), based on the primary clinical endpoint of years-of-life-lost (YLL) for mothers and infants. A probabilistic sensitivity analysis was used to assess uncertainty in the cost and clinical outcomes.

Results: The incremental per pregnancy cost of the intervention was USD\$12.66 (India), USD\$11.51 (Pakistan) and USD\$13.26 (Mozambique). As implemented, the intervention was not cost-effective, due largely to minimal differences in years-of-life-lost between arms. However, among women who received $\geq 8$ contacts ( 4 in Pakistan), the probability of health system and family (Pakistan) cost-effectiveness was $\geq 80 \%$ (all countries).

Conclusion: The intervention was likely to be cost-effective for women receiving $\geq 8$ contacts in Mozambique and India, and $\geq 4$ in Pakistan, supporting World Health Organization guidance on antenatal contact frequency.

Funding: The University of British Columbia, a grantee of the Bill \& Melinda Gates Foundation (OPP1017337).

Trial registration: clinicaltrials.gov. Registered 30 July 2013, https://clinicaltrials.gov/ct2/show/NCT01911494

\section{Contributions To The Literature}

- To date, health economic data relating to interventions for the hypertensive disorders of pregnancy have been based primarily on diagnostic and clinical interventions. Recent evidence suggests that community health worker-led initiatives can be cost-effective for maternal, neonatal and child health outcomes in low- and-middle-income countries (LMICs).

- Within the Community Level Intervention or Pre-eclampsia (CLIP) trials as implemented, we found no evidence of cost effectiveness overall, but increasing cost-effectiveness with a higher frequency of antenatal contact, particularly at least eight such contacts.

- Our findings support a role for community health workers in delivering the eight antenatal care contact model advocated by the World Health Organization.

\section{Introduction}

The hypertensive disorders of pregnancy are one of the primary global causes of maternal and fetal mortality (1) and morbidity, with pre-eclampsia specifically resulting in an estimated 76,000 maternal and 500,000 perinatal deaths annually (2). Recently, using data from the Community Level Intervention for Pre-eclampsia (CLIP) cluster randomised controlled trials (cRCTs) in India, Pakistan, Mozambique, and Nigeria, we estimated the incidence of pregnancy hypertension in developing countries to be at least $10 \%$, at least as high as in well-resourced settings (3).

Many of the pregnancy hypertension-related deaths occur either prior to women arriving at a health facility or after arriving there too late to prevent a fatal complication (4). As a result, there has been interest in mobilising frontline providers, such community health care workers (CHWs) to provide earlier care and facilitate referral to facility $(5,6)$. However, it has not been demonstrated that such an approach was effective or cost-effective.

The CLIP cRCTs in India, Pakistan, and Mozambique (NCT01911494) leveraged the existing CHW workforce to identify, implement initial treatment, and triage hypertensive pregnant women in their communities to facility, as well as to provide education to communities about obstetric emergencies and the hypertensive disorders of pregnancy specifically. There was no evidence that the CLIP intervention was effective in decreasing a composite of maternal and perinatal mortality and morbidity as implemented by the existing workforce. Consequently, the intervention was received by fewer women than anticipated (i.e., 7055 (90.0\%) in India, 11399 (56.3\%) in Pakistan, and 4809 (60.4\%) in Mozambique) (7-10). An a priori-determined contact-frequency analysis, indicated effectiveness in reducing maternal and perinatal mortality and morbidity among women who received the intervention per protocol; at least four visits in Pakistan, and at least eight visits in both India and Mozambique (7-10).

Health economic analyses in such global health trials are required, as they often provide policy direction for resource allocation for improving maternal and newborn health (11). Assessing cost-effectiveness of CHW-led interventions has been a matter of debate (12), and definitive determination of whether or not such an approach is a fiscally viable way of reducing adverse maternal and fetal outcomes is required.

The aim of this study was to assess the cost-effectiveness of the CLIP trial intervention in each of India, Pakistan, and Mozambique, and to determine whether or not cost-effectiveness differed between countries or according to the number of CHW-provided contacts a woman received.

\section{Methods}

This was a planned secondary analysis of the clinical and cost data collected from the CLIP cRCTs (NCT01911494) in India, Pakistan and Mozambique (13), based on a previously published protocol (14) and reported in line with a CHEERS checklist (15). (See Supplementary Appendix 1 for the Statistical Analysis Plan [SAP]). 


\section{Study population:}

The CLIP trials targeted pregnant women (15-49 years in India and Pakistan, and 12-49 years in Mozambique) across all intervention and control clusters (12 in each of India and Mozambique, and 20 in Pakistan). All women enrolled provided written consent. All were included in the economic analysis provided they had delivered by trial end and had data on the primary outcome of a composite of maternal and perinatal morbidity or mortality.

\section{CLIP Intervention:}

The methods for the CLIP trials are described in detail elsewhere. Briefly, each of the CRCTs aimed to reduce all-cause maternal and perinatal morbidity and mortality by community-level initial triage and treatment of hypertensive women in their community, and timely transfer to facility. The intervention combined community engagement and pregnancy hypertension-focussed home contacts by CHWs (i.e., accredited social health activists and auxillary nurse midwives (India), lady health workers (Pakistan), and agentes polivalentes elementares (Mozambique)). Community engagement meetings focussed on pre-eclampsia awareness and education around birth preparedness and complication readiness, and included the pregnant women themselves, as well as their family members and community stakeholders. CHW-led home contacts were centred around the use of the PIERS (Pre-eclampsia Integrated Estimate of RiSk)-On-theMove (POM) mobile health application for risk stratification (16). The POM application helped CHWs to respond to emergency conditions, take women's blood pressures, assess proteinuria (using dipsticks, at the first and any subsequently hypertensive visit), and use pulse oximetry (in Pakistan and Mozambique). For hypertensive women, the POM application directed $\mathrm{CHWs}$ to administer oral methyldopa, intramuscular magnesium sulphate, or refer to a comprehensive emergency obstetric care facility depending on the particular circumstance. In the control group, women received routine antenatal and postnatal care. In both control and intervention arms, relevant demographic, care-seeking, and clinical data were obtained at enrolment, through to 28 days postpartum (newborn) and six weeks (mother), through regular cross-sectional household surveys (every 3-6 months) and, in India, with additional facility record review.

\section{Costs:}

Within each country, detailed costs associated with the intervention were collected from annual budgets, receipts, and the number of intervention-related activities performed. Costs were divided into five main categories: 1) CHW training, 2) health worker incentives for providing POM visits, 3) drug administration costs resulting from POM visits, 4) community engagement sessions, and 5) supplies; for details, see Table S2. Total cost of the intervention is the sum of these five categories. Discounting of costs was not necessary due to the relatively short time period of the analysis. In the main analyses, we did not account for potential costs associated with differences in care-seeking (antenatally or postnatally) between intervention and control, as these measures were broadly similar between arms (8-10). In Pakistan, we included out-of-pocket costs to women, gathered by pilot tested focus-group discussions, in a separate analysis (Table S2). We did not account for the cost of trial surveillance in both arms, as a potential scale-up of this intervention would not incur these costs. Costs for each country were converted into USD rates based on the average exchange rate during the trial (i.e., India: 1 USD = 60 INR, Pakistan: 1 USD = 104.7 PKR, and Mozambique: 1 USD = 64.67 MZN).

\section{Outcomes}

The primary clinical endpoint was mortality for mothers and infants, including stillbirth and neonatal death. These rates were translated into years-of-life-lost (YLL) based on country-specific World Health Organization (WHO) life expectancies (17). In contrast to the CLIP primary composite outcome, pregnancyrelated morbidities for mothers and newborns were not included, as there are no validated years-of-life-disabled or disabilities associated with many of these outcomes in any of the three countries (18). Cost-effectiveness was summarised by incremental cost-effectiveness ratios (ICERs), which are interpreted as the average incremental cost relative to one year of life saved. That is, the cost that a policy maker would have to pay to extend life by one year.

\section{Study perspective:}

The cost-effectiveness analyses were based on a programmatic perspective, comparing the incremental cost of implementing the intervention both overall, and for various scenarios of POM-guided contacts delivered. This comparison assessed the cost to the health system of implementing the CLIP intervention at various levels but did not account for potential additional costs to women and their families, other than in a secondary analysis in Pakistan.

\section{Data analyses:}

The primary economic model used for the base case analysis and probabilistic sensitivity analysis was a decision-tree (19) based on the following possible decision points: i) arm of the trial; ii) number of POM contacts grouped into one of 0, 1-3, 4-7, or $\geq 8$ (within intervention arm branch); iii) whether or not a POMguided contact resulted in a referral to facility or use of either methyldopa or magnesium sulphate; and iv) outcome of pregnancy: maternal death, stillbirth, neonatal death or survival. See Figure S1 for details.

Within each country, the probability of each of these branches was estimated directly from the CLIP trial surveillance and POM databases. Standard errors for these probabilities were estimated and adjusted for the clustered structure of the data, and associated mean costs were estimated for each branch. Community engagement costs were assumed to be distributed evenly across the varying POM contact frequency groups. All other costs (CHW training and incentives, supplies, drugs) were distributed on a per contact basis, meaning branches on the tree corresponded to groups with more POM-guided contacts and associated costs, to appropriately estimate the associated implementation cost of each frequency ('scenario') of the intervention.

We conducted a standard probabilistic sensitivity analysis (20) to determine the uncertainty associated with our analyses. For this, probability and costs for each branch were simulated using the above parameters. Costs were assumed to have gamma distributions, outcomes were assumed to have beta distributions, and the probability of each number of POM visits was drawn from a Dirichlet distribution (Table S3). These simulations were run 10,000 times for each country and the results summarised with $95 \%$ credible intervals (as the $2.5^{\text {th }}$ and $97.5^{\text {th }}$ percentiles of the 10,000 runs) for the YLL, cost, and ICERs for 
each trial arm as well as at each intervention scenario. Results were also visualised using a cost-effectiveness plane (with points in the top, right corner considered to be cost-effective), and with willingness to pay curves and thresholds for each YLL averted (which shows for increasing cost to a decision maker the likelihood the intervention is cost-effective). Willingness to pay thresholds were based on three times the country-specific GDP/capita in 2016 (India = $\$ 5188$, Mozambique $=\$ 1287$, Pakistan $=\$ 4105)$.

All analyses were carried out using R Version 3.5.3.

\section{Results}

Data from 61,988 pregnancies of 69,320 enrolled in CLIP (89.4\%) were included in the CLIP economic analyses: 13,017 (88.1\%) from India, 35,791 (90.8\%) from Pakistan, and 13,810 (91.3\%) from Mozambique. The rates of maternal mortality, stillbirth, and neonatal death did not differ between trial arms in any of the CLIP trials, but women receiving at least eight POM-guided contacts in India and Mozambique, and at least four contacts in Pakistan, had lower rates of each of these outcomes, with stillbirth (India and Pakistan), neonatal mortality (Pakistan) being statistically significant, as previously reported (7-10).

Outcomes rates and trial intervention details can be found in Table 1.

Table 1

Main trial outcomes and intervention.

\begin{tabular}{|c|c|c|c|c|c|c|}
\hline & \multicolumn{2}{|l|}{ India } & \multicolumn{2}{|l|}{ Mozambique } & \multicolumn{2}{|l|}{ Pakistan } \\
\hline & Intervention & Control & Intervention & Control & Intervention & Control \\
\hline & $N=6908$ & $N=6109$ & $N=6941$ & $N=6239$ & $N=18441$ & $N=17350$ \\
\hline \multicolumn{7}{|l|}{ Trial outcomes } \\
\hline Composite maternal and perinatal outcome & $1252(18.1 \%)$ & $1157(18.9 \%)$ & $1246(18 \%)$ & $1172(18.8 \%)$ & $5373(29.1 \%)$ & $4187(24.1 \%)$ \\
\hline Maternal mortality & $7(0.1 \%)$ & $9(0.1 \%)$ & $15(0.2 \%)$ & $7(0.1 \%)$ & $55(0.3 \%)$ & $51(0.3 \%)$ \\
\hline Maternal morbidity & $371(5.4 \%)$ & $325(5.3 \%)$ & $735(10.6 \%)$ & $690(11.1 \%)$ & $2213(12 \%)$ & $1728(10.0 \%)$ \\
\hline Stillbirth & $191(2.8 \%)$ & $156(2.6 \%)$ & $196(2.8 \%)$ & $162(2.6 \%)$ & $935(5.1 \%)$ & $951(5.5 \%)$ \\
\hline Neonatal death & $179(2.6 \%)$ & $136(2.2 \%)$ & $218(3.1 \%)$ & $171(2.7 \%)$ & $1011(5.5 \%)$ & $962(5.5 \%)$ \\
\hline Neonatal morbidity & $813(11.8 \%)$ & $790(12.9 \%)$ & $275(4.0 \%)$ & $362(5.8 \%)$ & $2375(12.9 \%)$ & $1684(9.7 \%)$ \\
\hline \multicolumn{7}{|l|}{ Intervention } \\
\hline \multirow[t]{2}{*}{ Community engagement sessions } & 1379 group & - & 4243 group & 1379 group & 1368 group & - \\
\hline & & & & & 16,691 LHW-led & \\
\hline CHWs trained & 148 & - & 79 & - & 223 & - \\
\hline N POM guided contacts & 57,562 & & 26,145 & & & 54,782 \\
\hline POM guided contacts per pregnancy & $8.0[3.0,12.0]$ & - & $4.0[2.0,6.0]$ & - & $3.0[2.0,5.0]$ & - \\
\hline \multirow[t]{2}{*}{0} & 770 & - & $2796(40.3 \%)$ & - & 7905 & - \\
\hline & $(11.1 \%)$ & & & & $(42.9 \%)$ & \\
\hline \multirow[t]{2}{*}{$1-3$} & $1268(18.3 \%)$ & - & 936 & - & $2718(14.7 \%)$ & - \\
\hline & & & $(13.5 \%)$ & & & \\
\hline $4-7$ & $1363(19.7 \%)$ & - & $1818(26.2 \%)$ & - & $6008(32.5 \%)$ & - \\
\hline$\geq 8$ & $3507(50.8 \%)$ & - & $1391(20.0 \%)$ & - & $1810(9.8 \%)$ & - \\
\hline Pregnancies given methyldopa & $60(1.0 \%)$ & - & $28(0.7 \%)$ & - & $93(0.9 \%)$ & - \\
\hline Accepted & $51(85.0 \%)$ & - & $19(67.9 \%)$ & - & $92(98.9 \%)$ & - \\
\hline Pregnancies given $\mathrm{MgSO}_{4}$ & $67(1.1 \%)$ & - & $28(0.7 \%)$ & - & $103(1.0 \%)$ & - \\
\hline Accepted & $47(70.5 \%)$ & - & $13(46.4 \%)$ & - & 73 (70.9\%) & - \\
\hline Pregnancies referred to facility & $505(8.2 \%)$ & - & $263(6.3 \%)$ & - & $487(4.6 \%)$ & - \\
\hline Accepted & $401(86.7 \%)$ & - & $158(68.4 \%)$ & - & $305(83.6 \%)$ & - \\
\hline
\end{tabular}

Given the varying trial sizes, total implementation costs varied between countries; however, estimates of intervention costs per pregnancy were similar: USD\$13.0 in India, USD\$11.8 in Pakistan, and USD\$15.7 in Mozambique. Table 2 shows that the majority of the costs were related to POM-guided contacts and, therefore, costs were proportionately higher for women as they received an increasing number of contacts; the largest costs were for tablets and smartphones, and for the training of CHWs to deliver the intervention. In addition, costs in Pakistan were related to a much higher number of community engagement sessions. A detailed breakdown of costs can be found in Table S2. 
Table 2

Summary costs for intervention by country. All costs in USD.

\begin{tabular}{|llll|}
\hline & India & Mozambique & Pakistan \\
\hline Training of CHWs & 12755 & 53205 & \multirow{2}{*}{$79398^{*}$} \\
\cline { 1 - 3 } Incentives for delivering POM contacts & 39043 & 3097 & \\
\hline Methyldopa and MgSO $_{4}$ & 1365 & 7163 & 4864 \\
\hline Community engagement & 7048 & 9201 & 71942 \\
\hline Supplies & 29811 & 45383 & 60949 \\
\hline Total & 90022 & 108848 & 217153 \\
\hline Total per pregnancy overall & 13.0 & 15.7 & 11.8 \\
\hline 0 POM guided contacts & 1.01 & 1.35 & 3.91 \\
\hline 1-3 POM guided contacts & 3.51 & 9.66 & 9.56 \\
\hline 4-7 POM guided contacts & 8.06 & 22.10 & 17.48 \\
\hline$\geq 8$ POM guided contacts & 18.61 & 39.45 & 25.78 \\
\hline *Unable to be disaggregated from available data & & \\
\hline
\end{tabular}

In the base case analyses, the intervention was not found to be cost-effective in any of the three countries due to the lack of overall difference in maternal and perinatal mortality between arms (Table 3). Similarly, in the probabilistic analyses, fewer than $50 \%$ of samples were cost-effective in Mozambique and India, and $66 \%$ were cost-effective in Pakistan (Table S4). However, when disaggregating the intervention based on POM-guided contact frequency group, the groups receiving $\geq 8$ contacts (per protocol) showed cost-effectiveness in the base case in each country (ICERs $=43.3,48.7$, and 9.1 for each of India, Mozambique, and Pakistan respectively; in addition, the 4-7 contact group was cost-effective in Pakistan (ICER = 17.8). The cost-effectiveness plane (Fig. 1) depicts the results from the probabilistic sensitivity analyses; in each country, $>80 \%$ of the probabilistic samples were found to be cost-effective for the $\geq 8$ contact frequency group.

Table 3

Cost and YLL summaries (95\% credible interval from probabilistic sensitivity analyses) based on POM contacts received vs control arm. All da India program perspective Mozambique program perspective

Pakistan program perspect

\begin{tabular}{|c|c|c|c|c|c|c|c|c|c|c|c|}
\hline & \multicolumn{3}{|c|}{ India program perspective } & \multicolumn{4}{|c|}{ Mozambique program perspective } & \multirow[b]{2}{*}{ ICER } & \multicolumn{3}{|c|}{$\overline{\text { Pakistan program perspect }}$} \\
\hline & YLL & $\begin{array}{l}\text { Incremental } \\
\text { YLL }\end{array}$ & $\begin{array}{l}\text { Incremental } \\
\text { cost }\end{array}$ & ICER & YLL & $\begin{array}{l}\text { YLL } \\
\text { intervention } \\
\text { - control }\end{array}$ & $\begin{array}{l}\text { Incremental } \\
\text { cost }\end{array}$ & & YLL & $\begin{array}{l}\text { YLL } \\
\text { intervention } \\
\text { - control }\end{array}$ & $\begin{array}{l}\text { Increm } \\
\text { cost }\end{array}$ \\
\hline Control & $\begin{array}{l}3.36 \\
(2.97 \\
3.78)\end{array}$ & & - & - & $\begin{array}{l}3.25 \\
(2.65 \\
3.94)\end{array}$ & & - & - & $\begin{array}{l}7.63 \\
(7.16 \\
8.11)\end{array}$ & & - \\
\hline Intervention & - & & - & - & - & & - & - & - & & - \\
\hline Overall & $\begin{array}{l}3.74 \\
(2.68, \\
5.60)\end{array}$ & $\begin{array}{l}0.38 \text { (-0.82, } \\
2.28)\end{array}$ & $\begin{array}{l}13.0(2.29 \\
23.6)\end{array}$ & $\begin{array}{l}\text { Intervention } \\
\text { is } \\
\text { dominated* }\end{array}$ & $\begin{array}{l}3.67 \\
(2.42 \\
5.65)\end{array}$ & $\begin{array}{l}0.41(-1.00 \\
2.43)\end{array}$ & $\begin{array}{l}15.7(1.40 \\
40.05)\end{array}$ & $\begin{array}{l}\text { Intervention } \\
\text { is } \\
\text { dominated* }\end{array}$ & $\begin{array}{l}7.29 \\
(5.23 \\
9.26)\end{array}$ & $\begin{array}{l}-0.33(-2.39 \\
1.60)\end{array}$ & $\begin{array}{l}11.8(3 \\
23.41)\end{array}$ \\
\hline $\begin{array}{l}0 \text { POM } \\
\text { guided } \\
\text { contacts }\end{array}$ & $\begin{array}{l}3.41 \\
(2.52 \\
4.43)\end{array}$ & $\begin{array}{l}0.05(-0.94 \\
1.10)\end{array}$ & $\begin{array}{l}1.01(0.49 \\
1.73)\end{array}$ & $\begin{array}{l}\text { Intervention } \\
\text { is } \\
\text { dominated* }\end{array}$ & $\begin{array}{l}3.13 \\
(2.69 \\
3.62)\end{array}$ & $\begin{array}{l}-0.12(-0.93 \\
0.64)\end{array}$ & $\begin{array}{l}1.35(0.71 \\
2.12)\end{array}$ & $\begin{array}{l}11.25 \\
(-45.6 \\
45.7)\end{array}$ & $\begin{array}{l}7.65 \\
(7.04 \\
8.30)\end{array}$ & $\begin{array}{l}0.03(-0.76 \\
0.82)\end{array}$ & $\begin{array}{l}3.91(2 \\
5.25)\end{array}$ \\
\hline $\begin{array}{l}1-3 \text { POM } \\
\text { guided } \\
\text { contacts }\end{array}$ & $\begin{array}{l}4.53 \\
(3.02 \\
6.42)\end{array}$ & $\begin{array}{l}1.16(-0.43 \\
3.07)\end{array}$ & $\begin{array}{l}3.51(1.84 \\
5.77)\end{array}$ & $\begin{array}{l}\text { Intervention } \\
\text { is } \\
\text { dominated* }\end{array}$ & $\begin{array}{l}5.85 \\
(4.69 \\
7.12)\end{array}$ & $\begin{array}{l}2.60(1.28 \\
4.03)\end{array}$ & $\begin{array}{l}9.66(4.80 \\
16.18)\end{array}$ & $\begin{array}{l}\text { Intervention } \\
\text { is } \\
\text { dominated* }\end{array}$ & $\begin{array}{l}9.42 \\
(8.46 \\
10.43)\end{array}$ & $\begin{array}{l}1.80(0.72 \\
2.94)\end{array}$ & $\begin{array}{l}9.56(6 \\
13.59)\end{array}$ \\
\hline $\begin{array}{l}\text { 4-7 POM } \\
\text { guided } \\
\text { contacts }\end{array}$ & $\begin{array}{l}5.23 \\
(4.03 \\
6.68)\end{array}$ & $\begin{array}{l}1.87(0.58 \\
3.34)\end{array}$ & $\begin{array}{l}8.06(5.57 \\
11.02)\end{array}$ & $\begin{array}{l}\text { Intervention } \\
\text { is } \\
\text { dominated* }\end{array}$ & $\begin{array}{l}4.34 \\
(3.60 \\
5.16)\end{array}$ & $\begin{array}{l}1.09(0.08 \\
2.09)\end{array}$ & $\begin{array}{l}22.10 \\
(14.97 \\
30.52)\end{array}$ & $\begin{array}{l}\text { Intervention } \\
\text { is } \\
\text { dominated* }\end{array}$ & $\begin{array}{l}6.65 \\
(6.21 \\
7.11)\end{array}$ & $\begin{array}{l}-0.98(-1.63 \\
-0.31)\end{array}$ & $\begin{array}{l}17.48 \\
(12.72, \\
22.96)\end{array}$ \\
\hline $\begin{array}{l}\geq 8 \text { POM } \\
\text { guided } \\
\text { contacts }\end{array}$ & $\begin{array}{l}2.94 \\
(2.46 \\
3.45)\end{array}$ & $\begin{array}{l}-0.43(-1.07 \\
0.22)\end{array}$ & $\begin{array}{l}18.60 \\
(11.30 \\
28.2)\end{array}$ & $\begin{array}{l}43.28(-359 \\
426)\end{array}$ & $\begin{array}{l}2.44 \\
(1.89 \\
3.08)\end{array}$ & $\begin{array}{l}-0.81(-1.70 \\
0.07)\end{array}$ & $\begin{array}{l}39.45 \\
(26.15 \\
55.32)\end{array}$ & $\begin{array}{l}48.70(-155 \\
323)\end{array}$ & $\begin{array}{l}4.80 \\
(4.15 \\
5.48)\end{array}$ & $\begin{array}{l}-2.83(-3.64 \\
-1.99)\end{array}$ & $\begin{array}{l}25.78 \\
(21.49 \\
30.32)\end{array}$ \\
\hline
\end{tabular}

*Indicates that the intervention has no benefit in years of life lost

** Includes health system utilization costs from care-seeking reported by focus group

Note: all intervals are $95 \%$ credible intervals based of the probabilistic sensitivity analysis

The cost-effectiveness acceptability curves (Fig. 2) show the probability the intervention (for various scenarios) being cost-effective as a function of a decision-makers' willingness to pay to save one year of life lost (log scale). In each country, if a decision-maker was willing to pay three times the GDP per 
capita (red dashed line), the $\geq 8$ contact scenario (black solid line) has at least $90 \%$ certainty of cost-effectiveness. In Pakistan, at USD $\$ 400 / Y L L$, the probability of cost-effectiveness approaches near certainty (100\%), for both the $\geq 8$ and $4-7$ contact scenarios (red dashed line).

In Pakistan, the secondary analyses focussing on a societal perspective (including out-of-pocket costs to women and their families) yielded similar results to the primary analysis, although with slightly higher costs associated with each intervention contact frequency group, corresponding to slight increases in health care utilisation in the intervention arms. Details are in Table 3.

\section{Discussion}

\section{Principal findings:}

This study assessed the cost-effectiveness from the health system perspective, of the CLIP intervention in India, Pakistan, and Mozambique both overall and at various frequencies of POM-guided contacts per pregnancy. The cost of the intervention was very similar across each of the three countries on a per pregnancy basis $(\approx 12-16$ USD). As implemented, the intervention was not cost-effective overall in any of the three settings. However, maternal and perinatal mortality was significantly lower in women who received at least eight POM-guided contacts (four in Pakistan), and that scenario, the intervention had a high probability of cost-effectiveness in each country. In Pakistan, there were similar findings when including women's families' opportunity (out-of-pocket) costs for health system resource utilisation.

\section{Interpretation:}

Several recent reviews have summarised the current evidence on CHW-led initiatives (21-23), and many previous studies have identified cost-effectiveness for a variety of CHW-led interventions in reproductive, maternal, neonatal, and child health $(21,22)$, with further evidence supporting the use of integrated 'packages' of interventions (24). Despite this substantial body of information, many maternal health studies still do not include a health economic component (14), and those focussed on pre-eclampsia are based on diagnostic and clinical interventions, such as the use of magnesium sulphate (25,26). To our knowledge, this health economic analysis is the first to evaluate community-level interventions for pregnancy hypertension.

Our results are broadly consistent with prior findings, albeit requiring a frequency of contacts from $\mathrm{CHWs}$ that matches current WHO guidance.; indeed, the consistently observed decrease in maternal and perinatal mortality, and increase in cost-effectiveness, with at least eight POM-guided contacts provides external validation of the recent WHO recommendations for at least eight antenatal care contacts (27). Although the overall per-pregnancy costs were similar between countries, there was discrepancy in the cost of delivering at least eight POM-guided contacts, which was largely driven by differences in CHW training and incentive costs per-pregnancy. When considering at-scale implementation of interventions such as CLIP that require a high number of contacts to be effective, these cost differences would be important to consider, as CHW salaries and relative affordability differ between less-developed countries (28,29). That said, the relatively low cost in each country supports CHWs carrying out home-based visits during pregnancy in less-developed countries where interventions are urgently needed to reduce the burden of maternal and perinatal mortality.

\section{Strengths:}

Our study has several strengths. First, we prospectively collected both cost and outcome data within our clinical trial (11). Second, we implemented our intervention across three diverse settings in Africa and South Asia, and with a large sample size of $>60,000$ pregnant women overall; therefore, we believe that our findings are generalisable to other less-developed country settings. Further, the details of our budget allow for clear understanding of the cost of scaling any one or all components of the intervention. In the case of Pakistan, we were further able to corroborate our main findings by including data on out-of-pocket costs to women and their families, and, therefore, providing a broader societal perspective on the cost-effectiveness.

\section{Limitations:}

The main challenge associated with our economic analyses is that we were unable to use disability-adjusted-life-years (DALY) that include maternal and perinatal morbidity as an outcome measure. This is due to a lack of reliable DALYs for the collected morbidities in each of India, Pakistan, and Mozambique. Given that we consistently observed similar results in maternal morbidity (no effect overall, decrease with at least eight POM contacts) to mortality (7) it is probable that the cost-effectiveness results seen here would be similar if this could be included. On the other hand, survived neonatal morbidity was in some cases increased in the ${ }^{3} 8$ POM contact group, suggesting a trade-off between reduced mortality and increased survivable morbidity in neonates, which would require further exploration to determine severity as well as the values of women, health care providers, and their communities. Therefore, the inclusion of these data may have reduced the estimates of cost-efficacy seen here. Furthermore, given the scale of the trial and complexities of the health systems, reliable data on the out-of-pocket costs to women and health system costs associated with being referred were unavailable in India and Mozambique; this limits our conclusions to that of a health system perspective, rather than the preferred 'societal' approach in these two countries (30). In Pakistan, family opportunity cost data were available, and results were broadly similar to the programmatic perspective. In addition, we did not adjust costs for differences in routine antenatal care-seeking between arms as these measures and the associated cost were not available. Despite this, these measures were broadly similar between trial arms, and, therefore, we do not anticipate that this influenced the results (8-10). Finally, the decision-tree model used here could not adjust for differences between women who received a low and high number of POM visits that would have an impact on mortality, which may introduce a bias due to possible confounding. That being said, adjustment for such differences that were measured did not affect the association between increased visits and reduced outcomes $(7-10)$.

\section{Meaning:}


Depending on a decision maker's willingness to pay, having CHWs deliver home-based interventions at WHO-recommended frequency thresholds, is probably a cost-effective method for reducing maternal and perinatal mortality. The similarity in the cost of CHW visits and per-pregnancy trial costs overall between countries points to generalisability to other less-developed countries.

\section{Future work:}

As further emphasis is placed on increasing the number of antenatal contacts in less-developed countries, future studies should continue to assess the costeffectiveness of delivering these contacts, and possible integrated interventions by CHWs. Our data suggest that CHW-based interventions can be effective and cost-effective if a sufficient number of workers are available to deliver them at the required frequency. Future studies should estimate health system and out-of-pocket expenses in settings where families bare the cost of facility-based care. In addition, there is a need for continued development of 'years-of-lifedisabled' metrics for pregnancy-specific morbidities so that trials can better estimate the economic impact of their interventions.

\section{Conclusion}

While the CLIP intervention as implemented was not cost-effective at reducing YLL in any of the three countries, when implemented with at least eight POMguided contacts per pregnancy, there was both a reduction in YLL and a high probability of cost-effectiveness in each of the three CLIP countries. This supports the eight ANC-contact model advocated by the WHO.

\section{Declarations}

Ethics approval:

Ethical approval for the trials was granted by the University of British Columbia (UBC, H12-03497) and each country's relevant research ethics board (Aga Khan University, Pakistan, 2590-Obs-ERC-13; KLE University, India, MDC/IECHSR/2011-12/A-4, ICMR 5/7/859/12-RHN; and Centro de Investigação em Saúde da Manhiça (CIBS-CISM/038/14), Mozambique National Bioethic Committee (219/CNBS/14).

\section{Consent for publication:}

Not applicable.

\section{Availability of data and materials:}

The data on which the manuscript is based are freely available without restrictions from the CLIP Trials Data Access Committee, that can be contacted at PREEMPT@cw.bc.ca, as referenced on our website at https://PRE-EMPT.bcchr.ca.

\section{Competing interests:}

The authors have no competing interests to declare.

\section{Funding:}

The University of British Columbia, a grantee of the Bill \& Melinda Gates Foundation (OPP1017337).

\section{Author contributions:}

PvD designed the concept of the CLIP trials. ZAB and LAM were the co-principal investigators of the overall

CLIP trials project. MBB, ZAB, SSG, AAM, RNQ, KM, CS, and ES were co-principal investigators of the

individual CLIP trials. BAP, and MV co- ordinated the CLIP trials. JB conducted the analysis and wrote the first draft of the manuscript. The analysis plan was conceived by JB, ARK, CM and GJW. All authors read and approved the final version of the manuscript.

\section{Acknowledgements}

We thank the Government of Mozambique, Province of Sindh, and Government of India for their permission to integrate the CLIP trial into their health systems with in-kind support. We thank the families of the 144 women, 2591 fetuses, and 2677 neonates who died during the study period and who were willing to share their stories despite their grief. We particularly thank the following members of the Data Safety Monitoring Board: Romano

Nkumbwa Byaruhanga, Brian Darlow, Eileen Hutton (Chair), and Lehana Thabane. We also wish to acknowledge the entire CLIP trials working group (Table $\mathrm{S} 1$ ), comprised of all those who lead and took part in the planning, implementation and analyses of the trials. For general inquiries related to CLIP, please contact PRE-EMPT@cw.bc.ca. 


\section{References}

1. Steegers EAP, von Dadelszen P, Duvekot JJ, Pijnenborg R. Pre-eclampsia. In: The Lancet. 2010. p. 631-44.

2. Kassebaum NJ, Barber RM, Dandona L, Hay SI, Larson HJ, Lim SS, et al. Global, regional, and national levels of maternal mortality, 1990-2015: a systematic analysis for the Global Burden of Disease Study 2015. Lancet. 2016 Oct 8;388(10053):1775-812.

3. Magee LA, Sharma S, Nathan HL, Adetoro OO, Bellad MB, Goudar S, et al. The incidence of pregnancy hypertension in India, Pakistan, Mozambique, and Nigeria: A prospective population-level analysis. Myers JE, editor. PLOS Med [Internet]. 2019 Apr 12 [cited 2020 Mar 27];16(4):e1002783. Available from: http://dx.plos.org/10.1371/journal.pmed.1002783

4. Thaddeus S, Maine D. Too far to walk: Maternal mortality in context. Soc Sci Med. 1994;38(8):1091-110.

5. Haines A, Sanders D, Lehmann U, Rowe AK, Lawn JE, Jan S, et al. Achieving child survival goals: potential contribution of community health workers. Vol. 369, Lancet. Elsevier; 2007. p. 2121-31.

6. Firoz T, Sanghvi H, Merialdi M, Von Dadelszen P. Pre-eclampsia in low and middle income countries [Internet]. Vol. 25, Best Practice and Research: Clinical Obstetrics and Gynaecology. Bailliere Tindall Ltd; 2011 [cited 2020 Feb 21]. p. 537-48. Available from: http://www.ncbi.nlm.nih.gov/pubmed/21592865

7. Peter von Dadelszen, Zulfiqar A Bhutta, Sumedha Sharma, Jeffrey Bone, Joel Singer, Hubert Wong, Mrutyunjaya B Bellad, Shivaprasad S Goudar, Tang Lee, Jing Li, Ashalata A Mallapur, Khátia Munguambe, Beth A Payne, Rahat N Qureshi, Charfudin Sacoor, Esperanç and the CTWG. The Community-Level Interventions for Pre-eclampsia (CLIP) cluster randomised trials in Mozambique, Pakistan, and India: an individual participant-level meta-analysis. Lancet. 2020; In press.

8. Qureshia, Rahat N, Sana Sheikha, Zahra Hoodbhoy, Sumedha Sharma, Marianne Vidler, Beth A Payne, Imran Ahmed, J Mark Ansermino, Jeffrey Bone, Dustin T Dunsmuir, Tang Lee, Jing Li, Hannah Nathan, Andrew Shennan, Joel Singer, Domena Tu, Hubert Wong, Laura Ma ZB. Community level interventions for pre-eclampsia (CLIP) in Pakistan: A cluster randomised controlled trial. Pregnancy Hypertens. 2020;In press.

9. Bellad MB, Goudar SS, Mallapur AA, Sharma S, Bone J, Charantimath US, et al. Community level interventions for pre-eclampsia (CLIP) in India: A cluster randomised controlled trial. Pregnancy Hypertens. 2020 Jul 1;21:166-75.

10. Sevene E, Sharma S, Munguambe K, Sacoor C, Vala A, Macuacua S, et al. Community-level interventions for pre-eclampsia (CLIP) in Mozambique: A cluster randomised controlled trial. Pregnancy Hypertens. 2020 Jul 1;21:96-105.

11. Ramsey S, Willke R, Briggs A, Brown R, Buxton M, Chawla A, et al. Good research practices for cost-effectiveness analysis alongside clinical trials: The ISPOR RCT-CEA Task Force report. Value Heal. 2005;8(5):521-33.

12. Walker DG, Jan S. How do we determine whether community health workers are cost-effective? Some core methodological issues. Vol. 30 , Journal of Community Health. Springer; 2005. p. 221-9.

13. Payne B, Dadelszen P von, Bhutta Z, Magee L, Adetoro O, Sotunsa J. Protocol 13PRT/9313: the Community Level Interventions for Pre-eclampsia (CLIP) Trials: four prospective cluster randomised controlled trials comparing a package of interventions directed towards improving maternal and perinatal outcomes related to pre-ec. Lancet. 2018;

14. Khowaja AR, Mitton C, Bryan S, Magee LA, Bhutta ZA, von Dadelszen P. Economic evaluation of Community Level Interventions for Pre-eclampsia (CLIP) in South Asian and African countries: a study protocol. Implement Sci [Internet]. 2015 Dec 26 [cited 2019 Dec 6];10(1):76. Available from:

http://implementationscience.biomedcentral.com/articles/10.1186/s13012-015-0266-5

15. Husereau D, Drummond M, Petrou S, Carswell C, Moher D, Greenberg D, et al. Consolidated health economic evaluation reporting standards (CHEERS)explanation and elaboration: A report of the ISPOR health economic evaluation publication guidelines good reporting practices task force. Value Heal. 2013 Mar;16(2):231-50.

16. Payne BA, Hutcheon JA, Ansermino JM, Hall DR, Bhutta ZA, Bhutta SZ, et al. A Risk Prediction Model for the Assessment and Triage of Women with Hypertensive Disorders of Pregnancy in Low-Resourced Settings: The miniPIERS (Pre-eclampsia Integrated Estimate of RiSk) Multi-country Prospective Cohort Study. PLoS Med. 2014 Jan;11(1).

17. WHO | WHO Mortality Database. WHO. 2014;

18. James SL, Abate D, Abate KH, Abay SM, Abbafati C, Abbasi N, et al. Global, regional, and national incidence, prevalence, and years lived with disability for 354 Diseases and Injuries for 195 countries and territories, 1990-2017: A systematic analysis for the Global Burden of Disease Study 2017. Lancet. 2018 Nov 10;392(10159):1789-858.

19. Petrou S, Gray A. Economic evaluation using decision analytical modelling: Design, conduct, analysis, and reporting. BMJ. 2011 May 28;342(7808).

20. Methods for the Economic Evaluation of Health Care Programmes: Michael F. Drummond, Mark J. Sculpher, Karl Claxton, Greg L. Stoddart, George W. Torrance: 9780199665884: Health Policy: Amazon Canada.

21. Vaughan K, Kok MC, Witter S, Dieleman M. Costs and cost-effectiveness of community health workers: Evidence from a literature review [Internet]. Vol. 13, Human Resources for Health. BioMed Central Ltd.; 2015 [cited 2020 Feb 21]. p. 71. Available from: http://human-resources-

health.biomedcentral.com/articles/10.1186/s12960-015-0070-y

22. Costs and Cost-Effectiveness of Community Health Investments in Reproductive, Maternal, Neonatal, and Child Health [Internet]. [cited 2020 Feb 21]. Available from: https://www.mcsprogram.org/resource/costs-cost-effectiveness-community-health-investments-reproductive-maternal-neonatal-childhealth/

23. Pallas SW, Minhas D, Pérez-Escamilla R, Taylor L, Curry L, Bradley EH. Community health workers in low- and middle-income countries: What do we know about scaling up and sustainability? Vol. 103, American Journal of Public Health. American Public Health Association; 2013. p. e74. 
24. Adam T, Lim stephen S, Mehta S, Bhutta ZA, Fogstad H, Mathai M, et al. Cost effectiveness analysis of strategies for maternal and neonatal health in developing countries. BMJ. 2005 Nov 12;331(7525):1107.

25. Shmueli A, Meiri H, Gonen R. Economic assessment of screening for pre-eclampsia. Prenat Diagn. 2012 Jan;32(1):29-38.

26. Simon J, Gray A, Duley L. Cost-effectiveness of prophylactic magnesium sulphate for 9996 women with pre-eclampsia from 33 countries: Economic evaluation of the Magpie Trial. BJOG An Int J Obstet Gynaecol [Internet]. 2006 Feb [cited 2020 Feb 21];113(2):144-51. Available from:

http://www.ncbi.nlm.nih.gov/pubmed/16411990

27. WHO recommendations on antenatal care for a positive pregnancy experience.

28. Rogers E, Guerrero S, Kumar D, Soofi S, Fazal S, Martínez K, et al. Evaluation of the cost-effectiveness of the treatment of uncomplicated severe acute malnutrition by lady health workers as compared to an outpatient therapeutic feeding programme in Sindh Province, Pakistan 11 Medical and Health Sciences 1117 Public Health and Health Services. BMC Public Health [Internet]. 2019 Jan 17 [cited 2020 Feb 21];19(1):84. Available from: https://bmcpublichealth.biomedcentral.com/articles/10.1186/s12889-018-6382-9

29. Taylor C, Griffiths F, Lilford R. Affordability of comprehensive community health worker programmes in rural sub-Saharan Africa. BMJ Glob Heal. 2017;2(3).

30. Byford S, Raftery J. Perspectives in economic evaluation. BMJ. 1998 May 16;316(7143):1529.

\section{Figures}
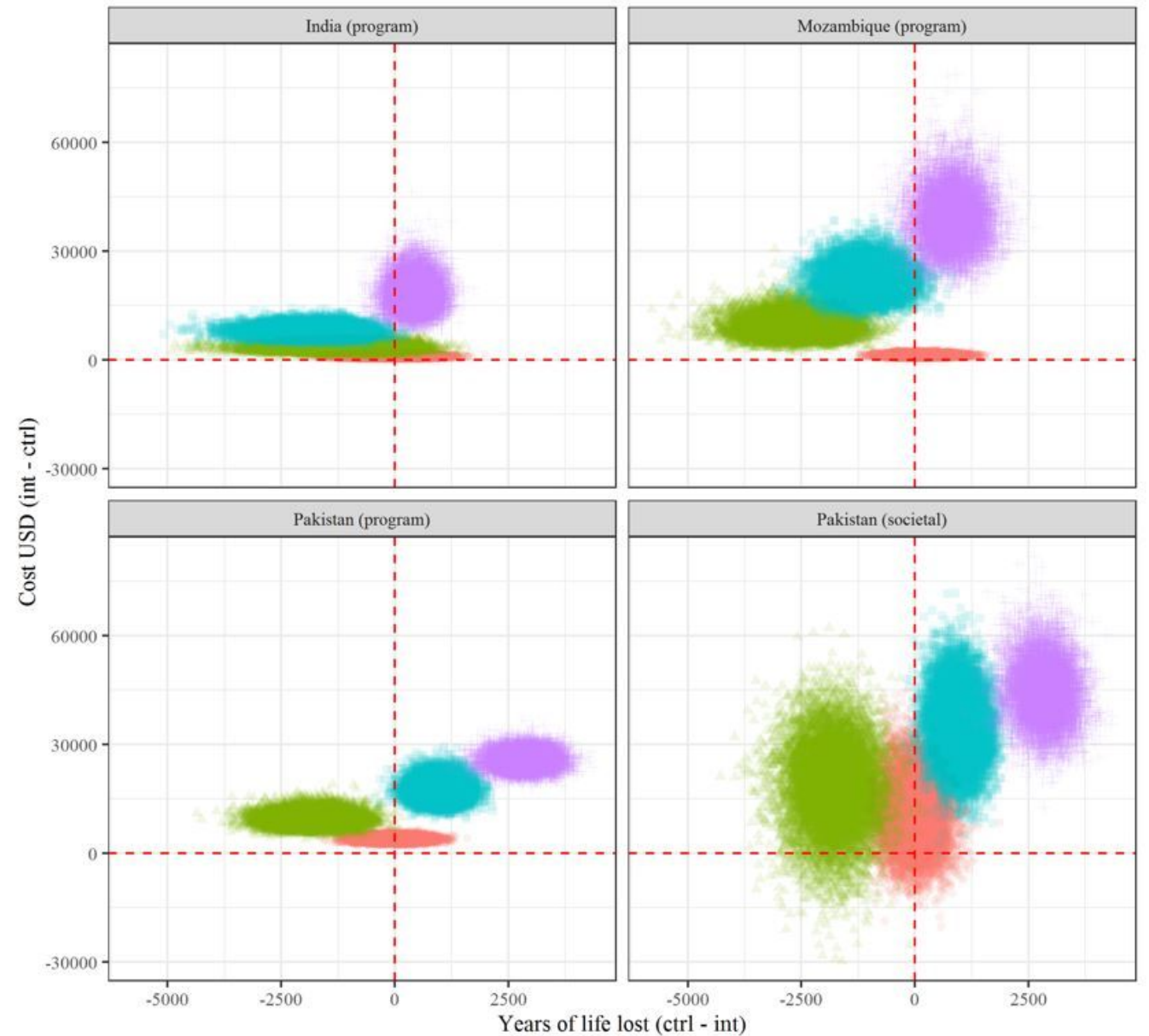

Number of POM contacts $\quad \begin{array}{llll}0 & 1-3 & 4-7 & \geq 8\end{array}$

\section{Figure 1}

Probabilistic sensitivity analyses of cost effectiveness by number of POM guided contacts received. Points in the north east quadrant are classified as cost effective. All data is per 1000 pregnancies. 


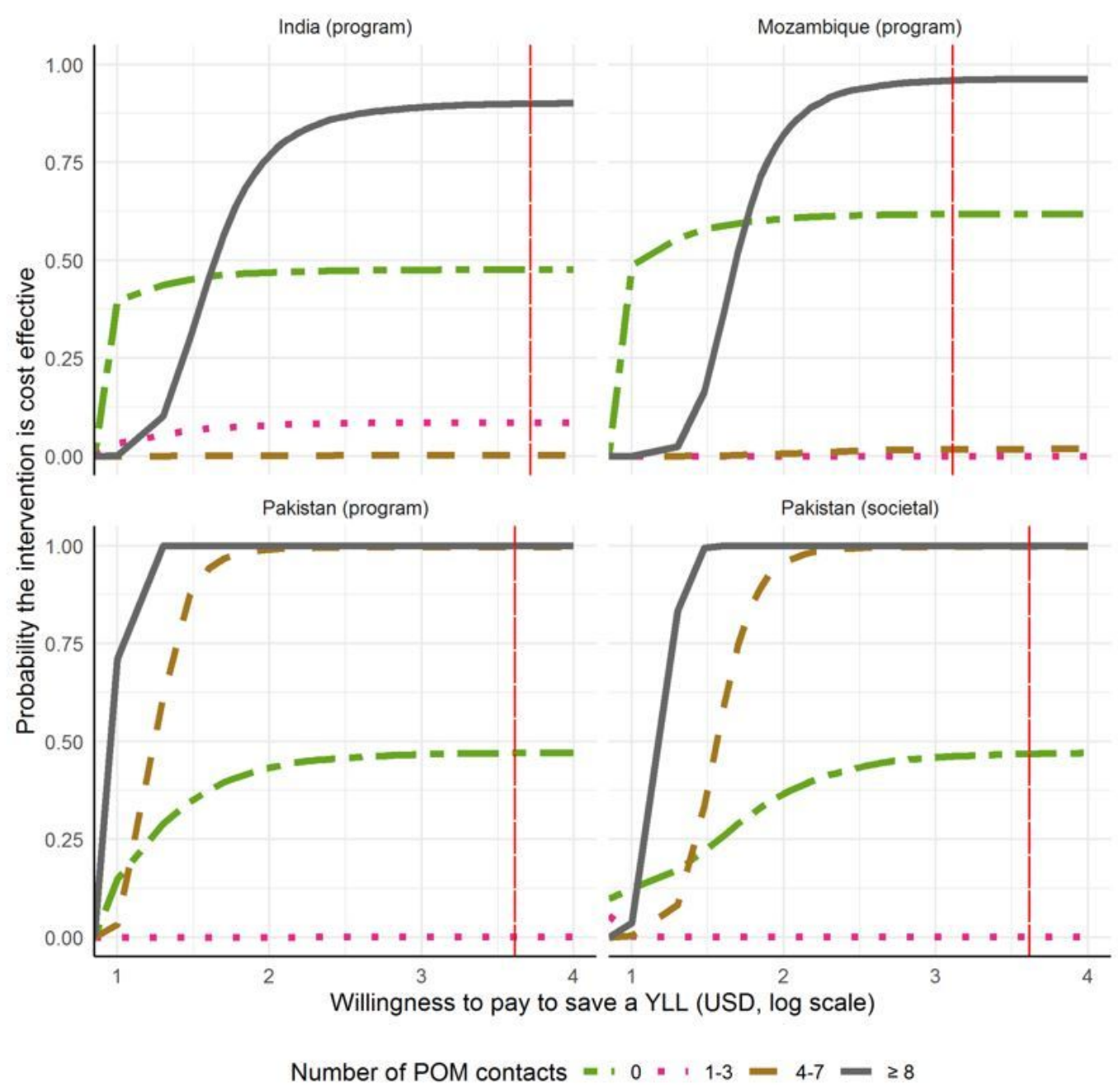

Figure 2

Cost effectiveness acceptability curves, by number of POM guided contacts received: probability that the intervention is cost effective as a function of a decision maker's willingness to pay to save one year of life lost. The vertical red line represents country-specific willingness to pay thresholds based on $3 x$ the GDP/capita.

\section{Supplementary Files}

This is a list of supplementary files associated with this preprint. Click to download.

- Supplementaryfiles08192020.docx

- CHEERSchecklist07142020.pdf 